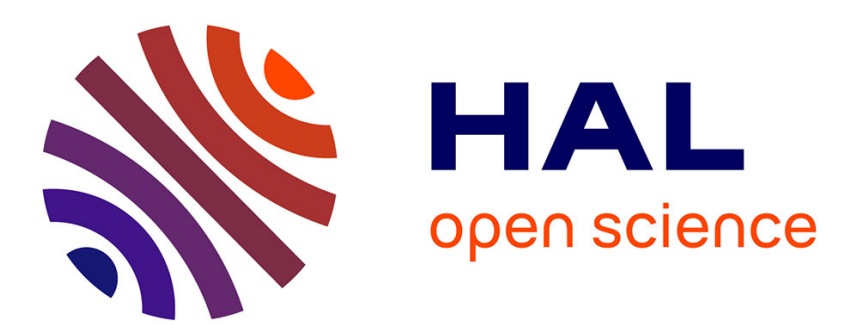

\title{
Toward an automatic segmentation of mitral valve chordae
}

Daryna Panicheva, Pierre-Frédéric Villard, Marie-Odile Berger

\section{To cite this version:}

Daryna Panicheva, Pierre-Frédéric Villard, Marie-Odile Berger. Toward an automatic segmentation of mitral valve chordae. SPIE Medical Imaging, SPIE, Feb 2019, San Diego, United States. pp.10953151095323, 10.1117/12.2511943 . hal-02050846

\section{HAL Id: hal-02050846 https://hal.inria.fr/hal-02050846}

Submitted on 27 Feb 2019

HAL is a multi-disciplinary open access archive for the deposit and dissemination of scientific research documents, whether they are published or not. The documents may come from teaching and research institutions in France or abroad, or from public or private research centers.
L'archive ouverte pluridisciplinaire $\mathbf{H A L}$, est destinée au dépôt et à la diffusion de documents scientifiques de niveau recherche, publiés ou non, émanant des établissements d'enseignement et de recherche français ou étrangers, des laboratoires publics ou privés. 


\title{
Toward An Automatic Segmentation Of Mitral Valve Chordae
}

\author{
Daryna Panicheva ${ }^{\mathrm{a}}$, Pierre-Frédéric Villard ${ }^{\mathrm{b}}$, and Marie-Odile Berger ${ }^{\mathrm{c}}$ \\ a,b, c Université de Lorraine, CNRS, Inria, LORIA, F-54000 Nancy, France
}

\begin{abstract}
Heart disease is the leading cause of death in the developed world. ${ }^{1}$ Cardiac pathologies include abnormal closure of the mitral valve, ${ }^{2}$ which can be treated by surgical operations, but the repair outcome varies greatly based on the experience of the surgeon. Simulating the procedure with a computer-based tool can greatly improve valve repair. Various teams are working on biomechanical models to compute the valve behaviour during peak systole. $^{3-5}$ Although they use an accurate finite element method, they also use a tedious manual segmentation of the valve. Providing means to automatically segment the chordae and the leaflets would allow significant progress in the perspective of simulating the surgical gesture for the mitral valve repair.

Valve chordae are generalized cylinders: Instead of being limited to a line, the central axis is a continuous curve. Instead of a constant radius, the radius varies along the axis. In most of the cases chordae sections are flattened ellipses and classical model-based methods commonly used for vessel enhancement ${ }^{6}$ or vessel segmenta$\operatorname{tion}^{7}$ fail. In this paper, we exploit the fact that there are no other generalized cylinders than the chordae in the micro CT scan and we propose a topology-based method for the chordae extraction. This approach is flexible and only requires the knowledge of an upper bound of the maximum chordae radius. Examples of segmentation are provided on three porcine datasets. The reliability of the segmentation is proved with a dataset where the ground truth is available.
\end{abstract}

Keywords: Topology-based image analysis, segmentation methodologies

\section{INTRODUCTION}

The mitral valve dysfunction is one of the common causes of cardiovascular diseases. ${ }^{2}$ The mitral valve separates the left atrium from the left ventricle providing one-way blood flow. A healthy valve is composed of two thin leaflets (stiff and flat structures) supported by tubular chordae tendineae (see figure 1, left). In the case of valve malformation the leaflets do not provide hermetic closure. In this case leaks may appear. The treatment of such diseases requires surgical intervention. Valve repair is preferred, as it gives better results than organ replacement. ${ }^{8}$ In order to improve repair outcome, patient-specific modeling of the valve behaviour is carried out. Computer simulations would allow the clinician to manipulate the model and thus to choose the technique which is best suited for the particular patient.

Image-based modeling includes segmentation of the valve components. Majority of existing models exploit a manual segmentation, which is tedious and varies greatly depending on the expert. Taking this into account, we focused on an automatic solution. In this paper, we propose a method to automatically segment the mitral valve chordae as a first step towards a fully automatic tool to extract the entire valve anatomy (see on figure 1 , left).

Contrary to many methods that rely on a model-based segmentation for tubular structures, ${ }^{6,7}$ we propose to locally classify structures in images as chordae or non-chordae depending on their topological properties.

The paper is organized as follows : the dataset and acquisition procedure are described in section 2 . The segmentation method is detailed in section 3. Eventually, the segmentation results for three datasets are presented in section 4 .

Further author information:

E-mail: daryna.panicheva@loria.fr, pierrefrederic.villard@loria.fr, marie-odile.berger@loria.fr 


\section{DATA ACQUISITION}

Six porcine hearts were scanned using high-resolution computed tomography (X-TEK HMXST225, now owned by Nikon Metrology). The mitral valve was acquired in the closed state with $120 \mathrm{~mm} \mathrm{Hg}$ of air pressure delivered through the aorta to simulate systole peak. A protocol was established to ensure high-quality micro CT scans. This included fixing the heart in a stable position and pressurizing it with air (figure 2). Scanner settings were established based on trial-and-error in order to avoid artifacts due to movement or drying/shrinking of the cardiac tissue during image acquisition. The number of scanning angles was 1600, the exposure time for each image was $500 \mathrm{~ms}$, the $\mathrm{x}$-ray tube power was set to $10.5 \mathrm{~W}$ with $\mathrm{U}=175 \mathrm{kV}$, and a $0.5 \mathrm{~mm}$ copper filter was used to achieve sufficient contrast and resolution. The result after reconstruction was a $2000 \times 2000 \times 2000$ 3D image with isotropic voxel resolution of $0.05 \mathrm{~mm}$, from which a region of interest for the mitral valve was selected. These scans produced high-contrast volumetric images mainly composed of air (Hounsfield density $\approx$ 1000) and soft tissue (Hounsfield density $\in[100,300]$ ).

a.
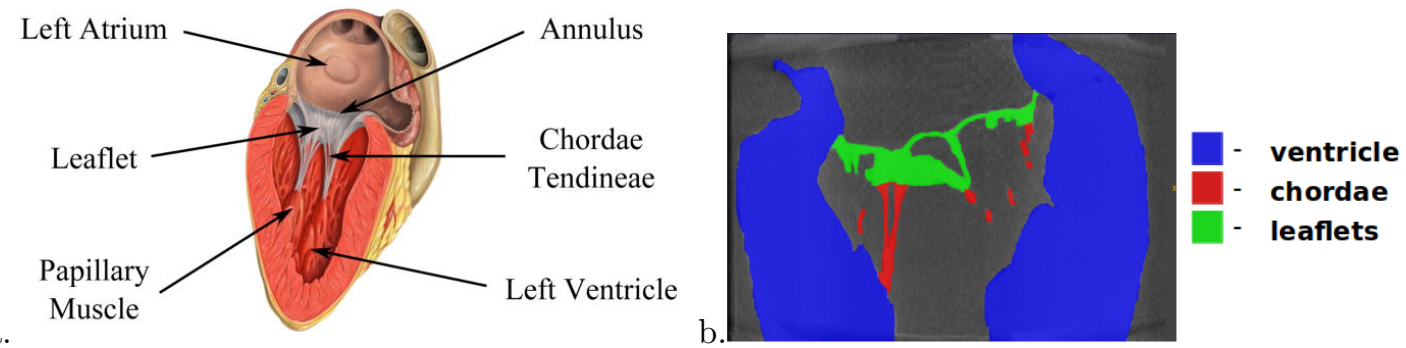

Figure 1. Mitral valve anatomy : a. Anatomy of the mitral valve (image from Patrick J. Lynch), b. Example of a sagittal view of the valve micro CT scan.
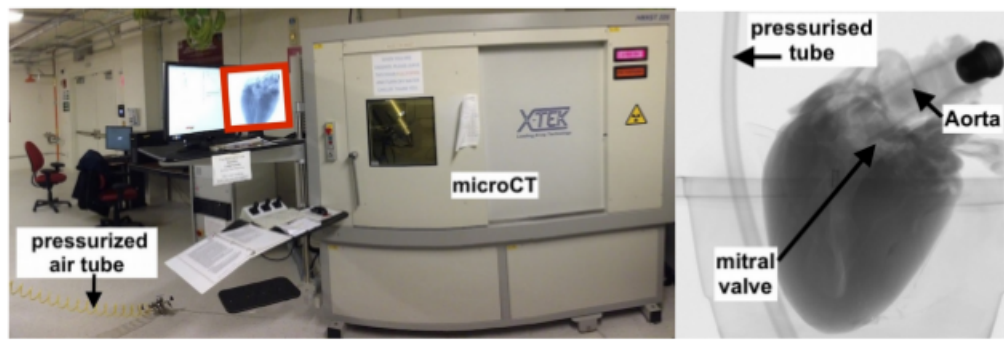

Figure 2. Data acquisition : Micro CT device with pressurized air tube going inside and its screen (in red and zoomed on the right) displaying an Xray of the porcine heart.

\section{METHOD}

\subsection{Topology-based segmentation of the chordae}

The soft tissues (chordae, leaflets and ventricle) appear as white structures in the micro CT scan due to the established experimental procedure. As can be seen on the manual segmentation slice illustrated on figure 1 (right), the junctions between chordae and leaflets are rather smooth and not distinct. Therefore, the main challenge consists in separating correctly the chordae from the other valve tissues. In addition to that, chordae topology is complex: the chordae are tubes of a variable section size containing numerous bifurcations. Given the foregoing, classical model-based methods of segmentation ${ }^{6,7}$ cannot be applied, since in our case, besides the target structures, the other objects (leaflets, ventricle and atrium) are present in the image and the contrast difference is not significant enough to distinguish them.

Since the chordae have elliptic non-regular sections, rather than a purely geometry-based approach, we propose a method that consists in analyzing the topological properties of structures present in the images. Hence, in the further steps we will exploit tissue contours extracted from the data. 
In the first stage, to minimize false defections, images are denoised with the nonlocal transform-domain filter. ${ }^{9}$ Then the tissue boundaries are extracted using the classical 3D edge detection procedure based on the Canny Deriche filter. ${ }^{10}$ The resulting set of points contains little noise. A surface mesh can be deduced from Poisson surface reconstruction ${ }^{11}$ in order to obtain the connectivity information between the points.

After the valve structure is represented in form of a mesh, mesh segmentation techniques are used to extract chordae. Topological constraints are commonly applied for mesh segmentation, but it often boils down to restrict each subregion to be topologically equivalent to a disk. ${ }^{12}$ In this work, we extract chordae components as features which are homeomorphic to a tube or to a bifurcation, whereas components homeomorphic to a disk are discarded.

As demonstrated in ${ }^{13}$ two compact surfaces are homeomorphic if and only if their boundaries have the same number of connected components (CC). Examples of surfaces with different topological characteristics are shown on figure 3.a. Blue ones have one $\mathrm{CC}$ and are equivalent to a disk, red ones have $2 \mathrm{CC}$ and are equivalent to a tube, green ones have $3 \mathrm{CC}$ and are equivalent to a bifurcation, black ones have $4 \mathrm{CC}$. It should be noted that there is no holes in the dataset meshes, so no additional connected components are created.

a.

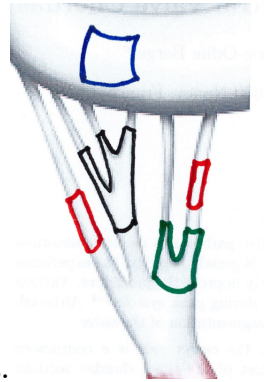

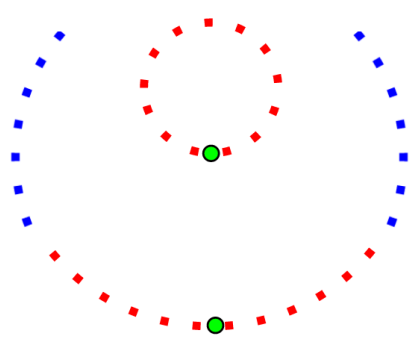

c.

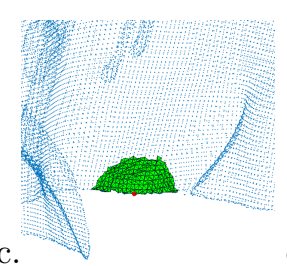

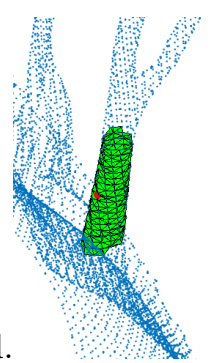

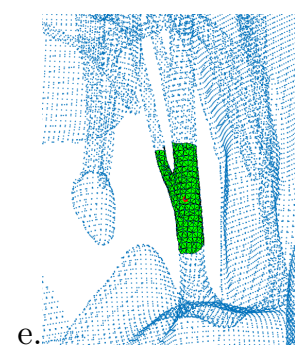

Figure 3. Topological characteristics of non-chordae, cylinder and bifurcation surfaces: a. Surfaces with different topological characteristics, b. Segmentation on synthetic data, c. Submesh corresponding to a non-chordae structure, d. Submesh corresponding to a tube, e. Submesh corresponding to a bifurcation.

As the chordae section is variable in form (ellipses with different eccentricity), in practice we use the knowledge of an upper bound of the maximum chordae radius $R$ as a parameter for the segmentation. The maximal chordae circumference can be estimated as $2 \pi R$. At each point $p$ of the mesh, we build a submesh centered on $p$ which contains points that are at a geodesic distance from $p$ less than $\pi R$. This allows to capture the whole tube section. Indeed, if $p$ belongs to a chordae, then the submesh should be closed and homeomorphic to a tube or a bifurcation. The submesh generation procedure is described in section 3.2.

The points are labeled depending on the number of CC in a submesh. Figure 3.b gives representative segmentation results on synthetic cases in dimension 1: for a tube the radius of which is less than $R$ (top), the red submesh generated from the green point is closed and is marked as chordae. For a point with a smaller local curvature (bottom), the generated submesh in red is not closed and the point is declared as non-chordae. Figures 3.c-e show the submesh computed for a real case for the point (in red). This point is identified as non-chordae in figure 3.c, as a tube in figure 3.d, and as a bifurcation in figure 3.e. The labeling process is detailed in section 3.3 .

It must be noted that we only need an upper bound of the radius and not an accurate value. This method is flexible since it detects tubes or bifurcations without requiring an explicit model of the section.

\subsection{Submesh extraction}

A submesh corresponds to the region enclosed by the iso-geodesic distance contour. In our case the distance is limited by semi-circumference of the largest chord. By definition, geodesic distance is the length of the curve between two points on the surface $x_{s}$ and $x_{e}{ }^{14}$ (see figure 4 .a) and the geodesic distance from the central point to each point in the set defines a geodesic distance map. An example on figure 4.b corresponds to a discrete case where color of the map indicates the proximity to the central point, red lines are iso-geodesic distance lines and yellow lines are examples of geodesic paths. ${ }^{14}$ Considering the size of our datasets ( $>300 \mathrm{~K}$ points), 
a.
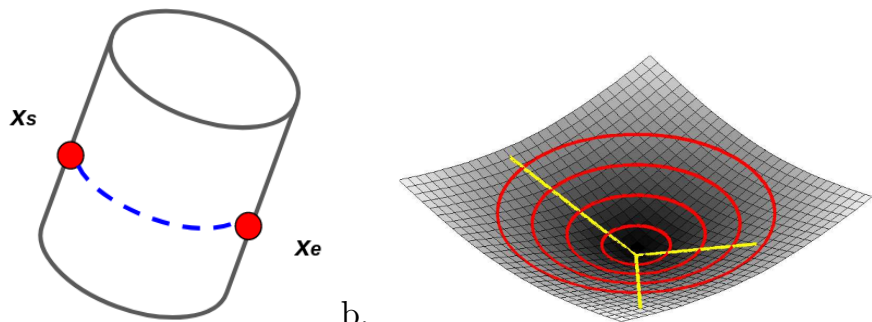

Figure 4. Geodesic paths : a. Geodesic path between two points on the surface, b. Geodesic distance map (in red iso-geodesic distance lines, in yellow - geodesic paths examples. ${ }^{14}$

the computation of a distance map for every point is hardly feasible in practice. Therefore, we propose an approximation scheme based on mesh connectivity. The submesh region is growing starting from the central point through its direct neighborhood (one-ring), on figure 5.a, the central point is in green and its direct neighbors are in red.

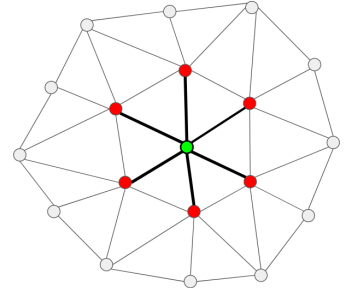

a.

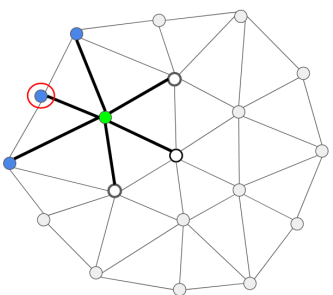

b.

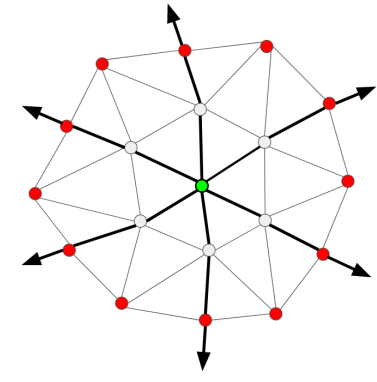

Figure 5. Mesh propagation scheme : a. Central point (green) and its one-ring (red), b. Choice of the closest point to the central one (circled in red) from the set of neighboring points (in blue), c. Approximated iso-geodesic line (red point) at the current propagation step, black arrows indicate the propagation paths directions.

The procedure goes as follows. Let $p s$ be an initial neighborhood (one-ring) of a central point $p_{c}$. We can limit the construction of the geodesic paths $P$ to $k$ directions defined by the direct neighbors $p s$ (black lines on figure 5.a). At each step of propagation in mesh the one-ring of every point $p_{i} \in p s$ is taken, excluding the points of the $p s$ and the central one $p_{c}$ in order to avoid the backward propagation (blue points on figure 5.b, noted $p s_{i}$ ). The point $p_{i+1}$ minimizing the euclidean distance $\left\|p_{i}, p_{i+1}\right\|$ is added to the path $P_{k}$ (circled in red on figure 5.b). The union of all the neighborhoods $p s_{i}$ forms new $p s$, which is an approximation of iso-geodesic line, see red points on the figure 5.c. The propagation continues till the distance to $p s$ (accumulated distances between the points in paths $P_{k}$ ) reaches $\pi R$. On figures 6.a-c initial, intermediate and final stages of mesh propagation are shown, lines are the approximated geodesic paths limiting the submesh points (in green). This approach also takes into account cases when mesh boundaries are present : as soon as a boundary is reached, propagation in this direction stops.

The output of the submesh extraction procedure is a set of points being connected by mesh to $p_{c}$ and laying on the distance smaller than $\pi R$ (green points on figure 6 ).

The geodesic distance map was calculated for extracted submeshes and the geodesic paths calculated from the distance map (line with black markers on figure 6.d) were compared to approximated paths resulting from the mesh (lines with asterisk markers on on figure 6.d). As well, obtained distances values where compared to those calculated from the distance map (see table 1 ). It can be seen that the mesh approximation approach gives less regular paths and thus the distances tend to be overestimated. Nevertheless, taking into consideration small difference in value (about 10,6\%) and the significant gain in computation time, this approximation is sufficient for the correct mesh extraction. 


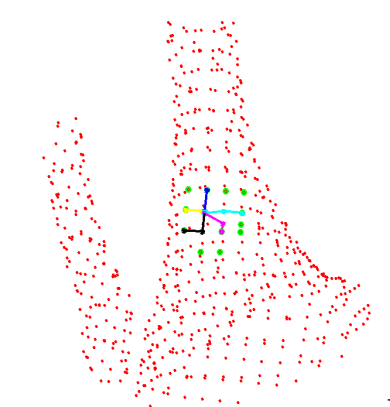

a.

b.

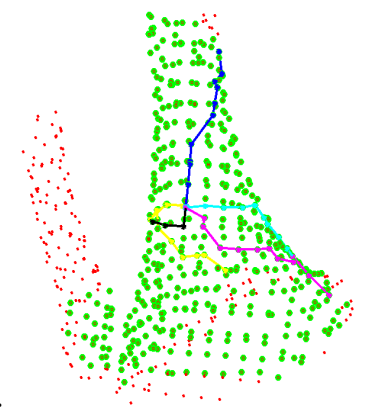

c.

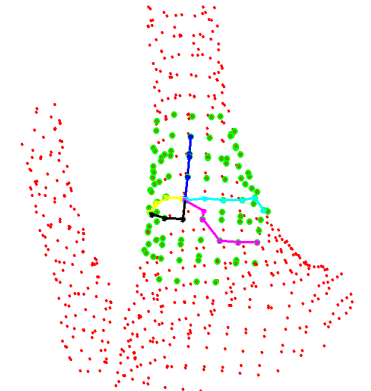

(1)

Figure 6. Computation of the submesh by mesh propagation (lines correspond to geodesic paths approximation, green points - to submesh points): a. Initial step of the algorithm, b. Intermediate step of the algorithm, c. Final step of the algorithm, d. Comparison of geodesic paths calculated from the distance maps (lines with black markers) with the paths resulting from the algorithm (lines with asterisk markers).

Table 1. Geodesic distance values

\begin{tabular}{|l|c|r|}
\hline Geodesic & Approximation & Relative difference, $\%$ \\
\hline 13,8427 & 16,0128 & 15,7 \\
13,5752 & 14,7587 & 8,71 \\
13,0107 & 13,3948 & 2,95 \\
13,8427 & 14,9095 & 7,7 \\
13,575 & 16,0479 & 18,2 \\
\hline
\end{tabular}

\subsection{Labeling procedure}

After the submesh centered on a point is extracted, the central point is labeled according to the number of submesh connected components. This procedure is repeated for all the vertices in mesh till all of them are labeled. The segmentation result is on figure 7.a, tubes are shown in red, bifurcations in green and multiple bifurcations are in magenta $(\# C C=4)$, blue corresponds to non-chordae structures. The separation between tubular structures and non-tubular ones is correct, as well as the bifurcation zones are well identified. However, this approach involves redundant calculations caused by the fact that submeshes of points being close to each other overlay, which considerably increases the computational time.

a.

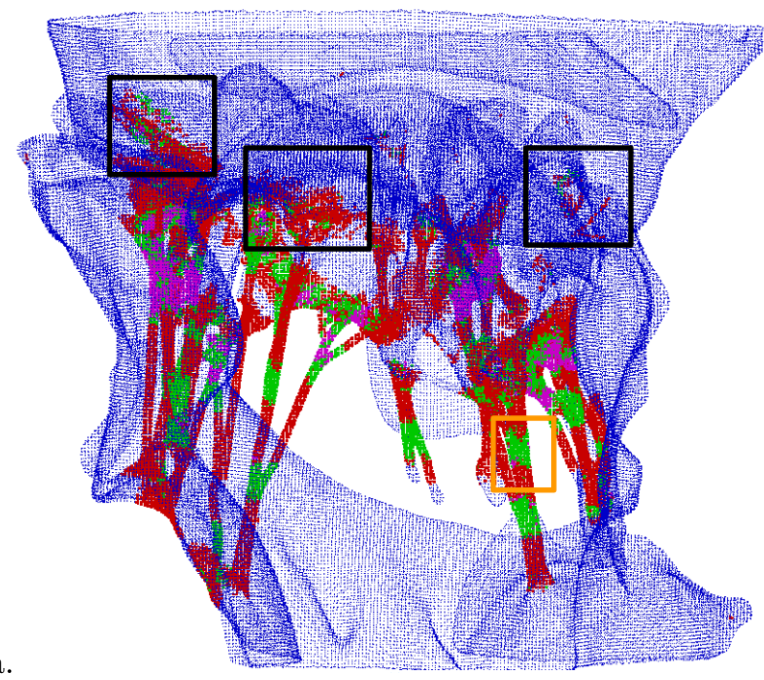

b.

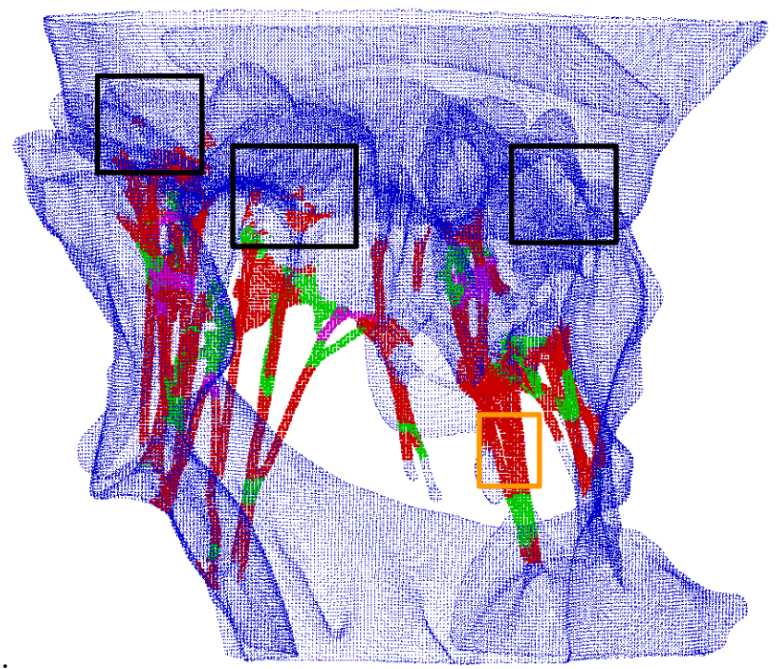

Figure 7. Segmentation results: a. Exhaustive approach (each point is labeled), b. Greedy approach (all points of submesh are labeled at once).

In order to reduce the computational time, we propose to label all the points of a submesh at once and not only the central one. Thus, the greedy procedure requires less iterations, but the overlays of submeshes still 
can occur and for points in such regions the labeling is repeated several times. To minimize the superposition of submeshes, a uniformly distributed sample of the points having no label yet is selected (red points on figure 8.a). This assures the spatial separation between submeshes corresponding to the sample points (see figure 8.b). The points of each submesh are labeled according to the following scheme: if point was not labeled yet it is assigned a label according to the \#CC in the current submesh, otherwise the existing label is overwritten if it is superior to the current one. In other words, the preference is given to a lower label. This allows to relabel the points in the overlaying zones, an example is illustrated on figure 8.c. First labeled as a bifurcation, points enclosed by rectangular are relabeled as a tube afterwords.

a.

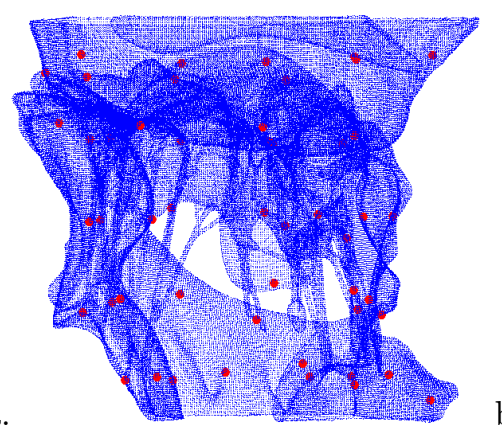

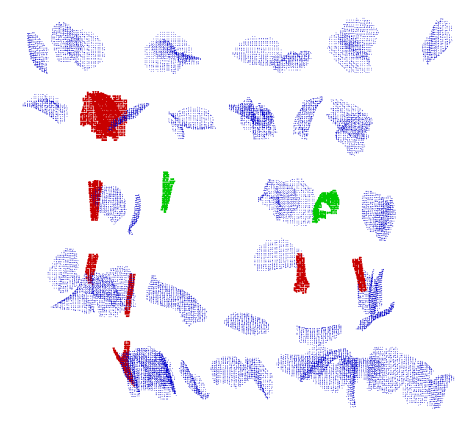

b.

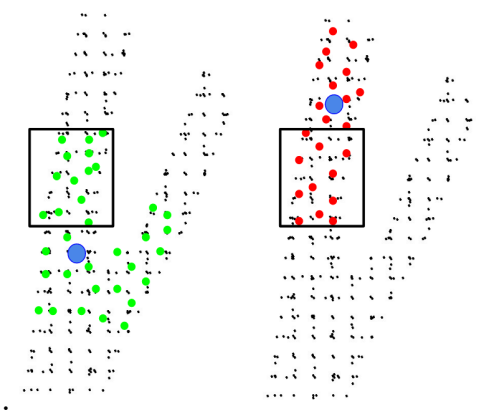

Figure 8. Labeling process : a. A uniformly distributed sample of central points for submesh extraction (red points), b. Labeled submeshes corresponding to the points of the uniformly distributed sample, c. Relabeling of the overlaying submeshes (central points in blue, overlaying zone in rectangular)

Segmentation results with the labels assigned during the greedy procedure are shown on figure 7.b, the color legend is the same as with exhaustive approach where submesh is extracted for each point (figure 7.a). When compared, both methods allow to separate correctly chordae and non-chordae structures. It must be noted that with the greedy approach number of false positives is decreased (see black zones on figure 7) since points from the overlaying regions are relabeled. On the contrary, the bifurcations are better identified with the exhaustive approach (see orange zones on figure 7). This can be explained by the section size variation of chordae in the data. In order to capture the large chordae, the maximal structure size is used as a parameter during mesh segmentation, which means that for smaller chords submeshes contain additional structures (see figure 9). Here the whole structure in the frame was captured, so the points of submesh were marked as a tube (having two CC) and not a bifurcation.

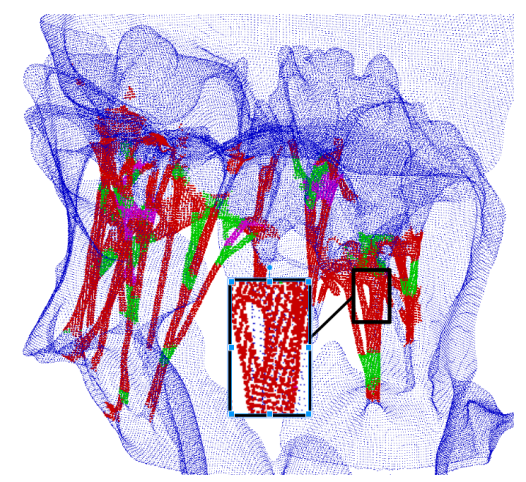

Figure 9. Segmentation of the chordae with labels.

\section{RESULTS}

The method has been tested on three micro CT scans. We have merged all the labels for chordae-type structures, the segmented chordae are shown in red on figure $10(\# C C \geq 1)$, blue color corresponds to non-chordae valve 

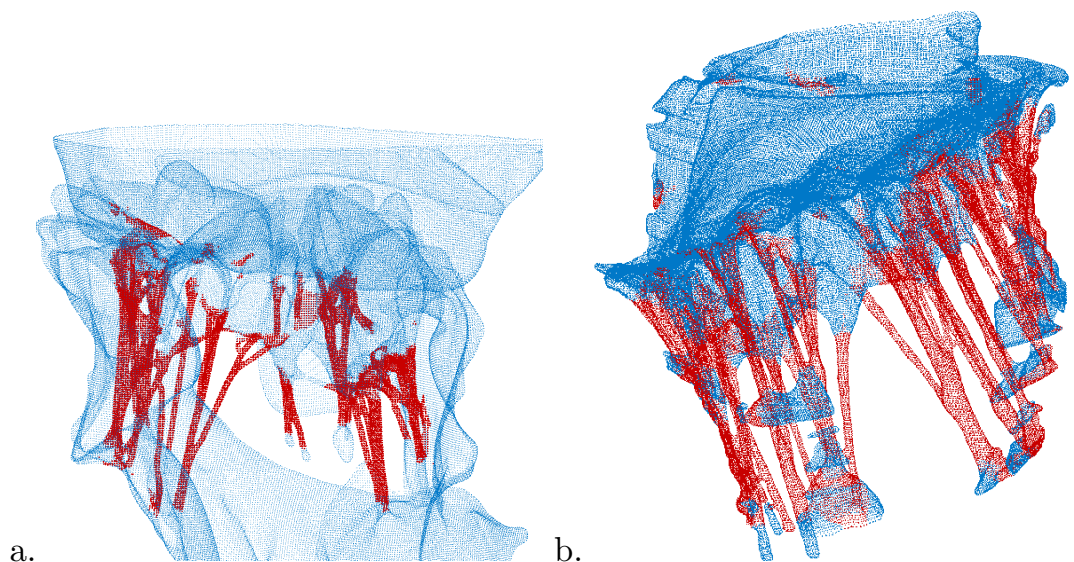

Figure 10. Segmentation of the chordae on 3 examples.
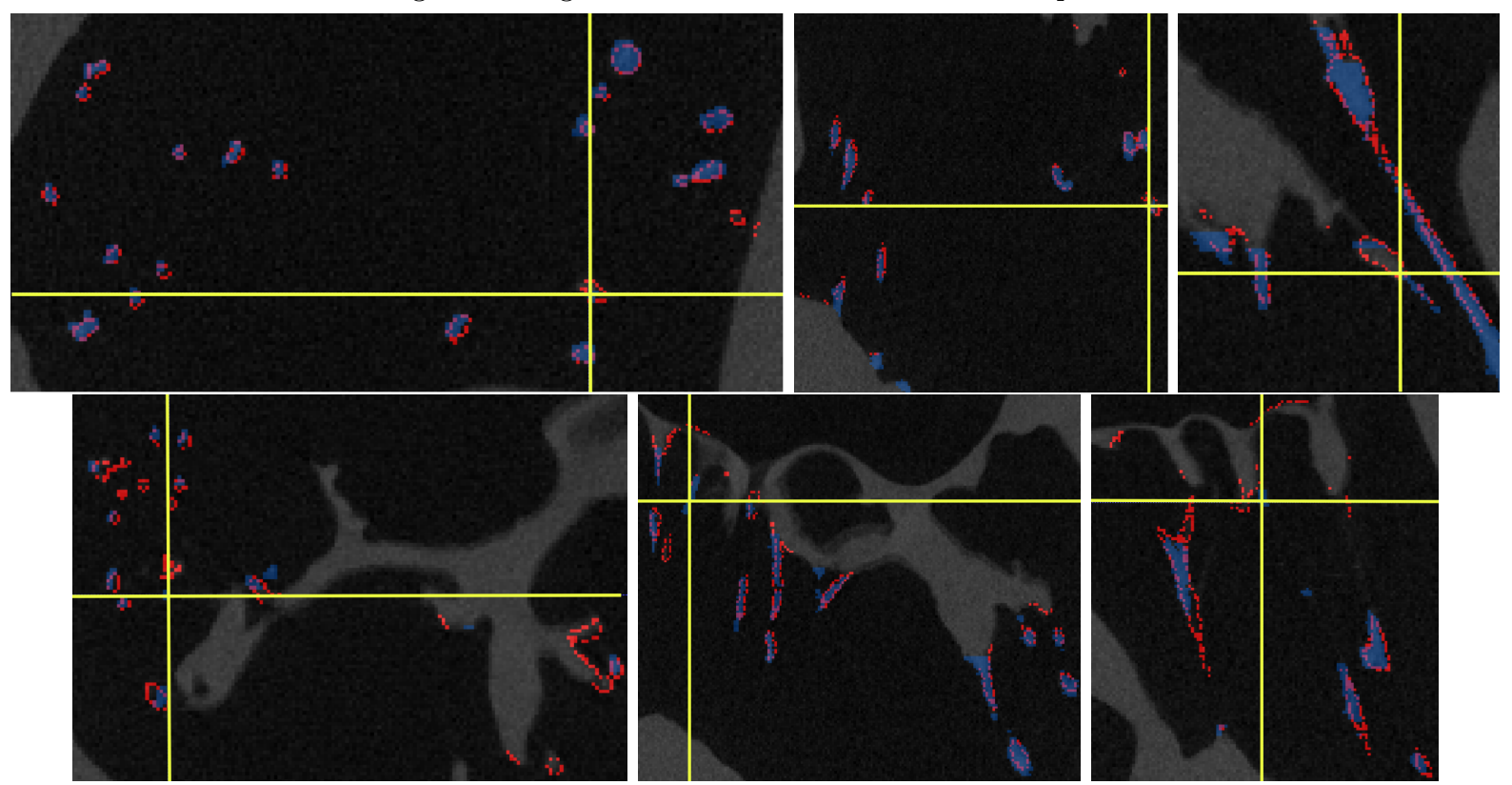

Figure 11. Superposition of the segmentation results (red) with the ground truth (blue) on axial coronal and sagittal views. First line: for a point at half maximum height of the chordae. Second line: for a point near the top of the chordae.

components. Overall, non-chordae structures are correctly identified and detected chordae ending points match up with actual chordae attachment points.

In order to have a quantitative evaluation of the method, we have compared the results of the algorithm with a ground truth segmentation done by the expert using ITKsnap. Manual segmentation of the micro CT scans is a tedious task (several hours are needed) and it is not always easy to distinguish the structures, so the results may vary depending on the expertise. We have superposed in figure 11 the results of our segmentation algorithm (in red) with the detection provided by the expert (in blue) on the axial (left), coronal (center) and sagittal (right) views. Two sets of views are provided for one point at half maximum height of the chordae (first line) and one near the top of the chordae (second line). As can be seen, all the chordae are detected at half height but some are missing or unduly detected in upper slices. The results are promising since the main structures of the chordae are correctly detected. 


\section{CONCLUSION}

A flexible method which only requires an upper bound of the maximum chordae size has been presented. The method is based on topological characteristics of the structures estimated locally in the mesh. For this task a strategy of submesh extraction was developed allowing to approximate fairly the geodesic paths and to reduce significantly the computational time comparing to the approach based on the geodesic map calculation.

The proposed approach was tested on three micro CT scans. It was shown that the method allows a proper segmentation of the chordae without requiring any parametric model of the section.

Further investigations will be dedicated to the use of a model-fitting approach in order to represent the extracted chordae in a form suitable for the mechanical model.

\section{ACKNOWLEDGMENTS}

This work was performed in part at the Harvard University Center for Nanoscale Systems (CNS), a member of the National Nanotechnology Coordinated Infrastructure Network (NNCI), which is supported by the National Science Foundation under NSF ECCS award no. 1541959.

\section{REFERENCES}

[1] "World Health Organisation cardiovascular disease." https://www.who.int/cardiovascular_diseases/ world-heart-day/en/. Accessed: 2018-12-18.

[2] Carpentier, A., Adams, D. H., and Filsoufi, F., [Carpentier's reconstructive valve surgery : from valve analysis to valve reconstruction], Elsevier (2010).

[3] Toma, M., Bloodworth, C. H., Pierce, E. L., Einstein, D. R., Cochran, R. P., Yoganathan, A. P., and Kunzelman, K. S., "Fluid-structure interaction analysis of ruptured mitral chordae tendineae," Annals of Biomedical Engineering 45, 619-631 (Mar 2017).

[4] Kong, F., Pham, T., Martin, C., Elefteriades, J., McKay, R., Primiano, C., and Sun, W., "Finite element analysis of annuloplasty and papillary muscle relocation on a patient-specific mitral regurgitation model," PLOS ONE 13, 1-15 (06 2018).

[5] Khalighi, A. H., Drach, A., Gorman, R. C., Gorman, J. H., and Sacks, M. S., "Multi-resolution geometric modeling of the mitral heart valve leaflets," Biomechanics and Modeling in Mechanobiology 17, 351-366 (Apr 2018).

[6] Frangi, A. F., Niessen, W. J., Vincken, K. L., and Viergever, M. A., "Multiscale vessel enhancement filtering," 130-137 (1998).

[7] Friman, O., Hindennach, M., Khnel, C., and Peitgen, H.-O., "Multiple hypothesis template tracking of small 3d vessel structures," Medical Image Analysis 14(2), 160 - 171 (2010).

[8] van der Merwe, J. and Casselman, F., "Mitral Valve ReplacementCurrent and Future Perspectives," Open Journal of Cardiovascular Surgery 9, 1179065217719023 (2017).

[9] Maggioni, M., Katkovnik, V., Egiazarian, K., and Foi, A., "Nonlocal transform-domain filter for volumetric data denoising and reconstruction," IEEE Transactions on Image Processing 22, 119-133 (Jan 2013).

[10] Canny, J., "A computational approach to edge detection," IEEE Trans. Pattern Anal. Mach. Intell. 8, 679-698 (June 1986).

[11] Kazhdan, M. M. and Hoppe, H., "Screened poisson surface reconstruction," ACM Trans. Graph. 32, 29:129:13 (2013).

[12] Shamir, A., "A survey on Mesh Segmentation Techniques," Computer Graphics Forum (2008).

[13] Georges, T., "The classification of surfaces with boundaries," tech. rep. (2011).

[14] Peyré, G., "Geodesic Methods in Computer Vision and Graphics," Foundations and Trends® in Computer Graphics and Vision 5(3-4), 197-397 (2009). 